\title{
The current landscape of 3D printing in oncological surgical interventions
}

\author{
Georgia Makin*,1 \\ ${ }^{1}$ Future Science Group, Unitec House, 2 Albert PI, Finchley, London N3 1QB \\ *Author for correspondence: g.makin@3dmednet.com
}

"The consensus in many medical fields is that the future of care lies in personalization. Therefore, 3D printing and associated technologies have immense opportunities for wide-spread adoption in research, clinical and surgical environments, particularly when considering complex tumor resection procedures."

First draft submitted: 7 August 2019; Accepted for publication: 7 August 2019; Published online: 19 August 2019

Keywords: 3D printing • surgery

In 2018, Cancer Research UK reported that 17 million new cases of cancer were diagnosed worldwide, with 9.6 million cases resulting in death [1]. Typical treatment strategies may involve surgery, chemotherapies and radiotherapies, but emerging technologies have paved the way for more personalized approaches such as immunooncology, targeted or hormone therapies.

The consensus in many medical fields is that the future of care lies in personalization. Therefore, 3D printing and associated technologies have immense opportunities for wide-spread adoption in research, clinical and surgical environments, particularly when considering complex tumor resection procedures.

Tailored pre-operative consultations, surgical technique rehearsals and bespoke solutions for instruments and implants are all ways in which 3D printing technologies have been disrupting oncological surgical workflows. Some claim that 3D models improve outcomes, reduce overall costs and positively impact the patient experience in and out of the operating room, as evidence from research into a wide range of pathologies suggests that $3 \mathrm{D}$ printing is a welcome assistant for surgical teams [2].

\section{Pre-operative consent}

A patient's journey through any planned surgical procedure begins with pre-operative informed consent. Under guidance from the Royal College of Surgeons of England (London, UK), a surgeon is required to ensure that a patient (or guardian) fully understands the procedure and any potential complications prior to signing a form indicating their consent to surgery [3].

The collection of 3D data from patients has long been possible through MRI and CT scanning technologies. Digital Imaging and COmmunications in Medicine (DICOM) data, derived from segmented versions of these scanned images, can be used to print replica, multi-material models capable of matching the weight, color and even consistency of a patient's internal anatomy. Landmark structures such as the ribcage, spine and organs can also be printed and incorporated into models, informing the position of tumors as well as reflecting relative size.

The physical handling of personalized models certainly has potential in facilitating opportunities for patients to ask questions and better understand explanations from members of the surgical team. An investigation into the use of 3D-printed personalized models with stage I lung cancer found that the use of models improved patient comprehension and informed consent for surgical resection of lung cancer tumors [4]. Patient knowledge was ranked significantly higher in questionnaires following consent consultations in a group exposed to half-scale models compared with groups without, leading the team to believe that the models improved patient understanding and therefore informed consent ahead of surgical procedures for this condition [4].

Future Medicine 
Personalized 3D-printed models could prove instrumental in developing an advanced and inclusive patient education experience: satisfying international consent guidelines, surgical teams and patients of all ages and backgrounds.

\section{Surgical planning \& rehearsal}

The same models as previously described can be repurposed to also educate and enhance the understanding of the surgeon, as surgical teams can ensure thorough preparation through rehearsal on accurate representations of the individual patient's anatomy.

Accurate models complete with vessels and internal anatomical landmarks can facilitate surgical team preparation, allowing for the anticipation of complications during surgery as well providing the opportunity for teams to refine technical skills and strategies in theatre. A study exploring the use of 3D-printed models in the pre-operative planning of laparoscopic partial nephrectomy in patients with renal tumors concluded that $3 \mathrm{D}$ printing provides an additional assistive tool for the performance of laparoscopic partial nephrectomy in patients with a RENAL score $\geq 8$, reporting an observed shortened warm ischemic time and less intra-operative blood loss when 3D-printed models were utilized [5].

In addition, investigation into the clinical value of $3 \mathrm{D}$-printed models for renal procedures and endoscopic colloid cyst resection established pre-operative models as highly valuable in the training and preparation of both junior and senior surgeons, due to the superior visualization of the anatomical networks $[6,7]$.

While it may not be practical to produce high quality pre-operative models for all surgical cases, the rehearsal of complex and rare cases appears an obvious first step towards the development and implementation of 3D printing in the operating room.

\section{Bespoke surgical instruments, tools \& guides}

An additional benefit of pre-operative rehearsal may be the identification of inadequate tools or instruments, particularly for rare or complex cases for which standard surgical equipment may not be suitable. It is possible for the team to design, develop and print their own tools to overcome anticipated challenges at a relatively low cost [8], reducing the risks of errors or added complications while the patient is under anesthetic.

Following 3D computer-assisted planning and even pre-operative rehearsal on a 3D-printed model, a surgical team may then have the capability to design a perfectly fitted cutting guide appropriate to the individual patient's 3D data. Dentists, for example, are already using chairside 3D printers to print better fitting crowns and drill guides utilizing techniques not dissimilar to those proposed for use by surgical teams for more invasive and complex surgical procedures $[9,10]$.

While bespoke, 3D-printed instruments could potentially allow access into hard-to-reach places, cutting guides are personalized, 3D-printed tools already used in orthopedic tumor resection procedures. Reducing soft tissue damage, time in theatre and even intra-operative surgeon decision-making [11,12], cutting guides appear to be popular subjects for case study analysis and useful additions to the orthopedic oncologist's surgical tool kit.

\section{Personalized implants}

The use of personalized implants to better fit individual patients is not a novel idea, but one that has been advanced and refined by the fine-detail capabilities of modern $3 \mathrm{D}$ printers and finishing techniques.

There are several companies worldwide offering personalized, 3D-printed solutions for implants for a range of orthopedic and soft tissue-based applications, employing a range of materials. A surgeon may, in the future, be able to print patient-specific implants using traditional medical-grade metal filaments, or could explore options adopting more porous materials, which may or may not involve regenerative medicine strategies for cell and tissue replacement and repair [13].

In mastectomy cases, for example, it may be possible to replace cancerous breast tissue with implanted scaffolds, which actively recruit cells into the area to repopulate the space with adipose tissue, based on success in studies involving rats [14]. Implants such as these may further be able to biodegrade, leaving behind regenerated and renewed tissue structures. Biodegradable polycaprolactone meshes and scaffolds have been successful in the surgical repair of orbital wall fractures [15] and cleft alveolus reconstruction [16] in human cases as well as tracheal replacement grafts in rabbits [17], indicating a versatile range of opportunities for adoption across various tumor types. 


\section{Conclusion}

It is clear that $3 \mathrm{D}$ printing is no longer regarded as a novel technology, nor a stranger to the field of medicine. With evidence supporting $3 \mathrm{D}$ printing as a valuable assistant for a wide range of surgical situations, it also seems there is very little $3 \mathrm{D}$ printing would struggle to assist a surgical team with.

There are, however, some challenges that need to be overcome to enable the transition of $3 \mathrm{D}$ printing into routine clinical care: a key one being cost. Despite the apparent low cost per 3D-printed object, the initial investment in building a $3 \mathrm{D}$ printing laboratory may still be too expensive to benefit all operating rooms and surgical environments. Tighter budgets may also struggle to accommodate further costs associated with training staff on new software, hardware and finishing techniques. Careful cost-benefit analysis would need to be carried out prior to implementing either insourced or outsourced 3D printing into a surgical workflow, considering different regulatory landscapes and a lack of published research for some pathologies and disease areas.

Within the field of oncology, the demand for personalized care from patient to patient, tumor to tumor and surgery to surgery is exceptionally high. A highly adaptable and personalization-friendly technology, 3D printing is positioned as a modern solution and affordable assistive tool in oncological surgical practice. As real-life case evidence builds in this area and confidence of use increases, the space for 3D-printed solutions in oncological surgical teams is expected to grow concomitantly, paving the way for a revolution in patient care and surgical practice.

3DMedLIVE 2019: 3D printing in surgery, the first conference from Future Oncology's parent publisher, Future Science Group, explores the benefits, challenges and solutions for the use of 3D printing for surgical applications. Experts from surgical teams, regulatory bodies and innovator organizations will join a unique programme of roundtables and case study presentations. Together, the event aims to convene the community of innovation and best practice to advance surgical applications of $3 \mathrm{D}$ printing. Accredited by the Royal College of Surgeons of England for up to 10.5 CPD points.

3DMedLIVE: 3D printing in surgery - 2-3 October 2019 - London, UK. www.3dmedlive.com

\section{Financial \& competing interests disclosure}

Georgia Makin is an employee of Future Science Group. The authors have no other relevant affiliations or financial involvement with any organization or entity with a financial interest in or financial conflict with the subject matter or materials discussed in the manuscript apart from those disclosed.

No writing assistance was utilized in the production of this manuscript.

\section{References}

1. Cancer Research UK. www.cancerresearchuk.org/health-professional/cancer-statistics/worldwide-cancer

2. Tack P, Victor J, Gemmel P, Annemans L. 3D printing techniques in a medical setting: a systematic literature review. Biomed. Eng. Online 15(1), 115 (2019).

3. Royal College of Surgeons of England. www.rcseng.ac.uk/standards-and-research/gsp/domain-3/3-5-1-consent/

4. Yoon SH, Park S, Kang CH, Park IK, Goo JM, Kim YT. Personalized 3D printed model for informed consent for Stage I lung cancer: a randomized pilot trial. Semin. Thorac. Cardiovasc. Surg. 31(2), 316-318 (2018).

5. Fan G, Meng Y, Zhu S et al. Three-dimensional printing for laparoscopic partial nephrectomy in patients with renal tumors. J. Int. Med. Res. doi:10.1177/0300060519862058 (2019) (Epub ahead of print).

6. Lupulescu C, Sun Z. A systematic review of the clinical value and applications of three-dimensional printing in renal surgery. J. Clin. Med. 8(7), 990 (2019).

7. Bodani VP, Breimer GE, Haji FA, Looi T, Drake JM. Development and evaluation of a patient-specific surgical simulator for endoscopic colloid cyst resection. J. Neurosurg. doi:10.3171/2019.4.JNS183184 (2019) (Epub ahead of print).

8. Spaas C, Lenssen O. Economic analysis of a low-cost virtual surgical planning protocol for mandibular reconstruction: a case series. Br J Oral Maxillofac Surg. doi:10.1016/j.bjoms.2019.06.017 (2019) (Epub ahead of print).

9. Derksen W, Wismeijer D, Flügge T, Hassan B, Tahmaseb A. The accuracy of computer guided implant surgery with tooth supported, digitally designed drill guides based on CBCT and intraoral scanning. A prospective cohort study. Clin. Oral Implants Res. doi:10.1111/clr.13514 (2019) (Epub ahead of print).

10. Dawood A, Marti Marti B, Sauret-Jackson V, Darwood A. 3D printing in dentistry. Br. Dent. J. 219(11), 521-529 (2019).

11. Xia RZ, Zhai ZJ, Chang YY, Li HW. Clinical applications of 3-dimensional printing technology in hip joint. Orthop. Surg. 11(4), 533-544 (2019). 
12. Wang B, Hao Y, Pu F, Jiang W, Shao Z. Computer-aided designed, three dimensional-printed hemipelvic prosthesis for peri-acetabular malignant bone tumor. Int. Orthop. 42(3), 687-694 (2018).

13. Visscher LE, Cheng M, Chhaya $\mathrm{M}$ et al. Breast augmentation and reconstruction from a regenerative medicine point of view: state of the art and future perspectives. Tissue Eng. Part B Rev. 23(3), 281-293 (2017).

14. Chhaya MP, Melchels FPW, Holzapfel BM, Baldwin JG, Hutmacher DW. Sustained regeneration of high-volume adipose tissue for breast reconstruction using computer aided design and biomanufacturing. Biomaterials. 52, 551-560 (2015).

15. Kim SY. Application of the three-dimensionally printed biodegradable polycaprolactone (PCL) mesh in repair of orbital wall fractures. J. Craniomaxillofac. Surg. 47(7), 1065-1071 (2019).

16. Kaye R, Goldstein T, Grande DA, Zeltsman D, Smith LP. A 3-dimensional bioprinted tracheal segment implant pilot study: rabbit tracheal resection with graft implantation. Int. J. Pediatr. Otorhinolaryngol. 117, 175-178 (2019).

17. Ahn G, Lee JS, Yun WS, Shim JH, Lee UL. Cleft alveolus reconstruction using a three-dimensional printed bioresorbable scaffold with human bone marrow cells. J. Craniofac. Surg. 29(7), 1880-1883 (2018). 